\title{
Knowledge Economy: Model Construction and Development Trend of Online Education
}

\author{
Junjie Liu1, Maxim Chernyaev²* \\ ${ }^{1}$ People's Friendship University of Russia RUDN University, Beijing, China \\ ${ }^{2}$ People's Friendship University of Russia RUDN University, Moscow, Russia \\ *Corresponding author: Maxim Chernyaev, chernyaev_mv@pfur.ru
}

Copyright: (C) 2022 Author(s). This is an open-access article distributed under the terms of the Creative Commons Attribution License (CC BY 4.0), permitting distribution and reproduction in any medium, provided the original work is cited.

\begin{abstract}
In regard to knowledge economy, the current concept in the model construction of online education, including distance education and online learning, generally refers to a kind of network-based learning behavior, similar to the concept of online training. Compared with traditional offline education methods, through the application of information technology and internet technology for content dissemination and rapid learning, online education has the characteristics of high efficiency, convenience, low threshold, and rich teaching resources. Online education covers a wide range of people, different forms of learning, and its classification methods are more diverse. Online education services are the fastest growing field of education informatization. At the moment, the most pressing problems include effectively integrating educational resources with internet technology, launching online education services and products that are highly interactive and would encourage personalized learning, increasing user stickiness, as well as avoiding trend-following and conceptualized investment.
\end{abstract}

Keywords: Knowledge economy; Model construction; Online education; SoLoMo; MOOC; Internationalization

Online publication: January 12, 2022

\section{Introduction}

The pressure from soaring rent and labor costs on the traditional offline education model and the individual needs of consumers have led to the gradual transformation of the teaching model to small classes and vertically integrated projects (VIP) courses. In this context, challenges, such as high costs and low profits, as well as difficulties faced by the training industry have become increasingly prominent. With the popularity of the internet and smartphones, mobile internet-related tools and network environments have greatly improved and upgraded.

Secondly, the limitations of the traditional education model are making the demand for personalized education more vigorous at this stage; in addition, the extension of education is gradually enlarging. Thanks to the national curriculum reform, local education infrastructure, and virtual campus construction, online education is generally considered to have broad development space. Especially during the pandemic, the value of online education as an important starting point for teaching reform has been highlighted.

\section{Method}

Upon experiencing the integration of shopping, tourism, and socialization with the internet, which also have broad market needs, education and healthcare have become the last two industries with a wide range of space and a certain degree of marketization. With the popularization of mobile communication 
infrastructure and the maturity of internet technology, the development of online education has accelerated. The high-speed bandwidth and 5G mobile era have greatly improved the possibility of live courses and fragmented time learning.

\section{Results}

\subsection{Development background}

\subsubsection{Government agencies encourage exploration}

Educational changes usually need to rise to the national level. From the perspective of online education, South Korea announced that paper-based textbooks would be abolished in 2015; in Japan, since 1998, 60 out of the 124 credits required for undergraduate graduation can be obtained through "distance teaching"; the former president of the United States had said that in the next four years, 99\% of American students will receive their education via the internet. In recent years, the Chinese government has also changed its attitude towards private education and attached importance to the application of information technology to achieve fairness in education and resource sharing.

In accordance with the strategic deployment of the "National Medium and Long-Term Education Reform and Development Plan (2010-2020)," China is building a national open university and has passed the formulation of the "National Open University Constitution." At the same time, the Ministry of Education is also actively promoting the construction of massive open online course (MOOC) content and has requested 985 colleges and universities to start offering such courses while granting corresponding course production subsidies ${ }^{[1]}$.

At the same time, the outline further clarified and put forward the concept of "education information network." From the policy to supporting the "education information network terminal" - the accelerated development of electronic schoolbags, the former State Administration of Press and Publication clearly included electronic schoolbags in the "12th Five-Year Plan" of the press and publishing industry, and the Ministry of Education took the lead in setting up a pilot e-schoolbag in Hongkou, Shanghai. The "Opinions on Promoting Information Consumption and Expanding Domestic Demand" and the "Notice of the State Council on Printing and Distributing the 'Broadband China' Strategy and Implementation Plan" further demonstrate the government's determination to support the development of the information industry and strengthen the development of the online education market heat.

\subsubsection{A large number of start-ups have emerged}

At this stage, start-ups involved in domestic online education are mainly concentrated in three areas: preschool education, K12 (referring to education from kindergarten to grade 12), and foreign language learning. According to Deloitte Research, preschool education applications account for about $60 \%$ of the 200 best-selling educational applications in China ${ }^{[2]}$.

This data shows that many Chinese parents nowadays use children's applications as tools for preschool education. The mentality of "do not let children lose at the starting line" has accelerated the explosion of preschool education applications and has also made preschool education the segment with the most distribution of online education start-ups.

Relative to preschool education, the K12 field is more complex, and its market potential is greater. It has also become the online education market segment that start-ups are most eager to compete for. However, the K12 field is different from the foreign language learning field that emphasizes on self-learning ${ }^{[3]}$. It involves multiple parties, such as students, teachers, and parents. Therefore, a product is required to complete closed-loop learning based on the needs of the above-mentioned three parties; no matter which party's needs are unaffected, full consideration or proper satisfaction will reduce user stickiness. 
From the perspective of product and service characteristics, online education start-ups can be divided into tool platforms, traffic platforms, question banks, online foreign teachers, online evaluations, vocabulary or spoken language learning, course schedules, and terminal tools. Typical enterprises in various categories are listed among them.

From the perspective of the main audience, white-collar workers and students under K12 education, who have strong demand for professional ability improvement, are still the mainstream groups that all parties pay the most attention to and try to compete for.

\subsubsection{Internet giants are in action}

Internet companies such as Baidu, Alibaba, and Tencent (B.A.T), the big three, as well as Qihoo, which are proficient in planning and layout, have also ventured into the field of education. Alibaba relies on Alipay to ensure trust in online education; Baidu relies on its core search advantages, and the acquisition of 91 mobile assistants has enhanced its mobile search capabilities; Tencent further uses WeChat to firmly socialize users. These internet giants have a deep understanding of user needs and a strong guiding role in user consumption behavior. Their entry into online education will attract the participation of many more groups. At the same time, the huge traffic of internet companies, such as B.A.T, also helps to solve the user and traffic problems of online education institutions.

Traditional internet giants and manufacturers focusing on education platforms have achieved significant traffic advantages and scale effects after long-term accumulation. However, educational needs are highly personalized, and the traffic is more special than other industries. Therefore, they have in-depth understanding of user needs and have achieved precise conversion.

\subsubsection{Traditional institutions consider the layout}

Online education is increasingly regarded as a trend in the future. While entrepreneurship is surging and giants marching in, traditional training institutions are also feeling the pressure of transformation.

For the K12 training field, New Oriental has launched a multi-port interactive platform based on personal computers (PCs), mobile terminals, and offline classrooms. Its 2014 fiscal year will continue to develop its interactive learning platform layout. Xueda Education will gradually realize SoLoMo ("social + localization + mobile") of its internal teaching resources and educational administration management system as well as offline business to online.

Traditional institutions can be said to be the most unique sequence in this battle. Compared with startups, traditional institutions have strong resource channels; compared with netizens, they have more abundant teaching content resources. However, the disadvantage of traditional institutions is that at the current stage, their online testing and offline expansion are sometimes trapped in a situation where they compete with each other ${ }^{[4]}$. At the same time, well-known traditional educational institutions are mostly listed companies, and their online layout requires them to face the issue of how to balance long-term investment and short-term shareholder returns.

\subsection{Development status}

At this stage, the parents of school-age students are more concentrated in the post-70s and post-80s groups, and their attitudes toward the internet are more open. However, for a long time, under the test-oriented and result-oriented teaching, student performance is still important for choosing and evaluating the quality of education. Online education still faces great challenges in ensuring learning effects and adapting to user needs ${ }^{[5]}$. 


\subsubsection{The online model is subjected to time competition and willingness to pay}

According to data from the Ministry of Education, as of December 31, 2012, there are 210 million primary and middle school students in China, and there is a huge market demand for K12 education ${ }^{[6]}$. Data shows that the size of the extracurricular tutoring industry in 2014 reached 32.5 billion US dollars, or about 200 billion yuan. In contrast, the current annual market share of online training is only about 300 million yuan, and the gap between online and offline markets is approaching thousand times.

Although the internet can bring certain price advantages due to the irreversibility of K12 children's growth and the high cost of trial and error, families are not sensitive to the price of education. "Education consumption" accounts for seven percent of the income of middle-class families in China. In addition to that, $54 \%$ of families spend more than 2,000 yuan on extracurricular tutoring each month.

It should also be noted that the extracurricular time of K12 students in China is very limited, and online education has to take hold of the effective time of students with offline institutions ${ }^{[7]}$. Education is a service system that attaches great importance to results. It is impossible for parents to disregard the guarantee of learning effects just to save road traffic jams, class registration fees, and other non-core factors.

\subsubsection{A lack of suitable content for online education}

In the early days of discussing about online education, many people used to compare China and the United States, but the biggest difference between the two is that the digital teaching environment in the US is richer in content and has a more mature supporting service system in addition to hardware.

The major listed companies in the US education category are higher education institutions. The main income of the famous Washington Post comes from the education business of Copeland. In addition to its universities, it is the world leader in Chartered Financial Analyst (CFA) exam content and has well-known educational content, such as Becker, ETS, Provider, etc.

However, China's private education itself has grown from the low end of the industrial chain. As examination standards are formulated by the government as a "one size fits all," domestic intellectual property rights have long been insufficiently protected. The content of Chinese education companies is basically based on examination materials research and foreign introduction. They lack scale and the ability to develop content independently. This made the emergence of online education in such a way that although hardware tools can make rapid progress, but the soft content has been slow to keep up.

The learning methods, paths, habits, etc. of online education are different from traditional education. The online model of moving offline content to online has been proven to be unable to meet user needs. However, due to the long-term weak content development capabilities, there is a shortage of both internet and education talents. There is a clear difference between the scattered areas of education and content development, in which content development has become a bottleneck restricting the development of online education $^{[8]}$.

\subsubsection{The profit model is still difficult to clarify, and the teaching achievement evaluation and conversion rate are challenges}

The internet has long been a free model in China. Recently, the "100 Education" under YY announced that TOEFL (Test of English as a Foreign Language) and IELTS (International English Language Testing System) courses will be offered to students for free, marking the start of the free battle. However, recalling that this model is not the first attempt in the education field, the initial promotion of New Oriental Education is to attract students with free lectures, and then partly convert into paying students. The efficiency of internet conversion and payment of online education is even more critical. 
Compared with the user stickiness and value-added charges of the internet, the long-term stickiness of education courses is still a big challenge. If interest courses, social networking, shopping, etc. can be regarded as frequent needs, education products appear relatively phased, and the continuity use by users will be its biggest test; teaching results become the main measurement indicators in exam courses. For online education that lacks compulsory learning constraints and sufficient content guarantees, it is even more difficult for the evaluation of teaching results to be objective and reasonable.

\section{Discussion}

It is worth thinking about it calmly. Although the current concept of online education is trending and the enthusiasm for capital injection is extremely high, the actual user usage and payment are not ideal. There are only a handful of profitable ones, and the scale of the network has not yet appeared.

Online education is a change in educational methods. Considering only from the perspective of technology and mode, China is not lagging behind with its current level. The gap lies mainly in the students' awareness of active learning and payment. However, the market opportunity does not come from the social consciousness to cater to the product; alike the "trust" obstacle of e-commerce in the early years, the opportunity will not be given to people who chant, "Everyone be honest," but rather, a solution similar to Alipay needs to be proposed.

\subsection{Serviceability}

The long-term problems faced by educational institutions are to a certain extent caused by the separation of purchasers and users. Parents, as the main decision-makers, hardly participate in the entire teaching process. The internet can effectively strengthen the communication between schools and parents, improve teaching effects through the combined efforts of family education, and meet the demand for information asymmetry. The main business of All-Tone Education, "School News Communication," is a product launched under this background. Its main function is to realize the information exchange between parents and schools.

\subsection{0}

Online education would eventually move toward a model where basic knowledge is free and value-added services are charged. Modes such as smart question banks, online answers, as well as learning and discussion communities are emerging, and some functions of offline institutions can be realized through the online scale. This can greatly liberate the offline manpower of organizations, promote efficiency and effectiveness, as well as increase customized value-added services.

\subsection{Socialization}

Group size and internal communication are very important to users' long-term continuous contribution. A successful platform does not only require a large group, it needs to have a certain degree of communication, so that the pursuit of prestige within the group or other motivations that help contribute can be stimulated, so as to realize user production and dissemination of content.

\subsection{MOOC}

Educational resources are still scarce, and in the long run, elite colleges and universities can provide academic education through open online courses. In higher education, since the threshold of their own examinations has made a fixed distinction for students, this makes it easy to accept a "slightly different" 
education with relatively less tuition.

\subsection{Content provider}

The internet platform has provided the convenience of content presentation. The industrial chain is gradually improving and maturing. The division of labor between content, platform, and channel vendors is clear. In the future, content vendors would become the core of the industrial chain. Of course, an important prerequisite is the improvement of China's intellectual property protection environment.

\subsection{Vocational education}

In the past ten years, vocational education focused on fields that involve skill training, such as culinary and auto repairs. With the upgrading of industries and fierce competitions in the job market, high-degree vocational training for on-the-job personnel is increasingly valued by the state, enterprises, and institutions. In addition, the innate advantages of the professional population in paying ability and willingness to learn are very suitable for online learning. In the context of future policy support and the increased demand, the development of related fields is worthy of attention.

\subsection{Application software}

Various types of application software products in the education field under the mobile internet are complicated. From the perspective of types, there are mainly four categories.

(1) Based on the needs of fragmented learning on mobile phones, such as word memorizing and question bank products.

(2) Based on the convenient voice use of mobile terminals, such as speaking and listening practice products.

(3) Animation and game products based on child users.

(4) Value-added promotional products based on the services of teaching institutions.

\subsection{Internationalization}

The essence of online education companies is still education, so whether it is the supply side-educational resources or the demand side-user resources, it is the core of the industry's competition for major companies. On the supply side, global educational resources are used as a whole.

With the rapid coverage of overseas users through investment, mergers and acquisitions is in fact an efficient model commonly used in the industry. Previously, NetDragon acquired $100 \%$ of Prometheus at a price of 130 million US dollars. This is a company that provides interactive teaching solutions with a combination of software and hardware. It was listed in the United Kingdom and has a business map covering more than 100 countries, 1.3 million classrooms, with users comprising of 2 million teachers and 30 million students worldwide. Through this acquisition, NetDragon has achieved great progress in internationalization, which is equivalent to directly covering 110 countries.

\section{Conclusion}

In conclusion, with highly developed informatization today, the capitalization, diversification, complexity, and internationalization of online education have become a trend, but the profit model is not clear enough; even in the fastest growing field of education informatization, the result of rapid expansion affects users' learning concepts and consumption habits, which would naturally attract more attention from practitioners 
and venture capital companies. The most urgent measure now is to effectively integrate educational resources and internet technology, launch online educational services and products that are highly interactive, encourage personalized learning to increase user stickiness, as well as avoid following trends and conceptualized investment.

\section{Disclosure statement}

The authors declare that there is no conflict of interest.

\section{References}

[1] Ministry of Education, 2010, Outline of China's National Plan for Medium and Long-term Education Reform and Development (2010-2020), National Medium and Long-term Education Reform and Development Plan Outline Working Group Office, Beijing, 21-22.

[2] Wang X, 2020, Analysis Report on the Development Status of Online Education in China. Industry Information Network, 2020(5): 7.

[3] Liu L, 2017, K12 Education Industry Analysis Report. Education Industry Research Institute, 2017: $10-15$.

[4] Ma Y, Li B, 2017, Online Education Industry Market Prospects and Investment Research Report. iResearch, 2017(5): 33-36.

[5] Chen X, 2005, Several Methods and Thoughts on the Construction of Distance Education Resources in Primary and Secondary Schools "China Educational Technology and Equipment." Longyuan Journal Network, 2005(3): 25-30.

[6] The State Council, 2015, Guiding Opinions on Actively Promoting the "Internet +" Action, Bulletin of the State Council of the People's Republic of China, 2015(40), Paragraph 3-5.

[7] Hao M, Qiao Z, 2017, Curriculum Teaching Reform and Practice in Vocational Colleges Under the Background of "Internet +" Education. China Vocational and Technical Education, 12(3): 23.

[8] Ma Z, Xin H, 2018, “Internet + Education” Research Literature Review. Industry and Technology Forum, 17(1): 15-17.

Bio-Byword Scientific Publishing remains neutral with regard to jurisdictional claims in published maps and institutional affiliations. 